Draft Version November 1, 2018

Preprint typeset using $\mathrm{LATE}_{\mathrm{E}} \mathrm{X}$ style emulateapj v. 08/22/09

\title{
THE END OF AMNESIA: A NEW METHOD FOR MEASURING THE METALLICITY OF TYPE IA SUPERNOVA PROGENITORS USING MANGANESE LINES IN SUPERNOVA REMNANTS
}

\author{
Carles Badenes $^{1,2}$, Eduardo Bravo ${ }^{3}$ and John P. Hughes ${ }^{4}$ \\ Draft Version November 1, 2018
}

\begin{abstract}
We propose a new method to measure the metallicity of Type Ia supernova progenitors using Mn and $\mathrm{Cr}$ lines in the X-ray spectra of young supernova remnants. We show that the $\mathrm{Mn}$ to $\mathrm{Cr}$ mass ratio in Type Ia supernova ejecta is tightly correlated with the initial metallicity of the progenitor, as determined by the neutron excess of the white dwarf material before thermonuclear runaway. We use this correlation, together with the flux of the $\mathrm{Cr}$ and $\mathrm{Mn} \mathrm{K} \alpha$ X-ray lines in the Tycho supernova remnant recently detected by Suzaku (Tamagawa et al. 2008) to derive a metallicity of $\log (Z)=$ $-1.32_{-0.33}^{+0.67}$ for the progenitor of this supernova, which corresponds to $\log \left(Z / Z_{\odot}\right)=0.60_{-0.60}^{+0.31}$ according to the latest determination of the solar metallicity by Asplund et al. (2005). The uncertainty in the measurement is large, but metallicities much smaller than the solar value can be confidently discarded. We discuss the implications of this result for future research on Type Ia supernova progenitors.
\end{abstract}

Subject headings: stars:binaries:close — supernova remnants — supernovae:general — X-rays:ISM

\section{INTRODUCTION}

Despite decades of continuing effort, the nature of the progenitor systems of Type Ia supernovae (SNe) remains unknown. The most widely accepted theoretical scenarios involve the thermonuclear explosion of a $\mathrm{C}+\mathrm{O}$ white dwarf (WD) destabilized by accretion of material from a binary companion, either another WD (double degenerate systems, DDs) or a normal star (single degenerate systems, SDs). Observations of supernova rates in distant galaxies suggest at least two different ways to produce Type Ia SNe (Scannapieco \& Bildsten 2005): a 'prompt' channel associated with young stellar populations and a 'delayed' channel associated with old stellar populations. At present, it is not clear what relationship, if any, these channels have with the theoretical scenarios, or even if the proposed scenarios can yield Type Ia $\mathrm{SNe}$ at the observed rate (MaOz 2008). Most attempts to constrain the fundamental properties of the progenitors have been unsuccessful, including direct identification in optical pre-explosion images (Maoz \& Mannucci 2008), searches for $\mathrm{H}$ stripped from the binary companion in SN spectra (Leonard 2007), and searches for prompt emission from circumstellar interaction (Panagia et al. 2006; Hughes et al. 2007). Very recently, the possible identification of the progenitor in a pre-explosion X-ray image of SN 2007on by Voss \& Nelemans (2008) has been questioned by post-explosion images (Roelofs et al. 2008). Campaigns to look for the surviving companion star to the exploded WD in the nearby Tycho supernova

\footnotetext{
${ }^{1}$ Department of Astrophysical Sciences, Princeton University. Peyton Hall, Ivy Lane, Princeton NJ 08544-1001; badenes@astro.princeton.edu

2 Chandra Fellow

${ }^{3}$ Departament de Física i Enginyeria Nuclear, Universitat Politècnica de Catalunya, Diagonal 647, Barcelona 08028, Spain; and Institut d'Estudis Espacials de Catalunya, Campus UAB, Facultat de Ciències, Bellaterra, Barcelona 08193, Spain; eduardo.bravo@upc.es

${ }^{4}$ Department of Physics and Astronomy, Rutgers University. 136 Frelinghuysen Rd., Piscataway NJ 08854-8019; jph@physics.rutgers.edu
}

remnant (SNR), known to be of Type Ia origin, have also produced controversial results (Ruiz-Lapuente et al. 2004; Ihara et al. 2007). This lack of clues to the identity of the progenitors in the observations of Type Ia SNe is sometimes referred to as 'stellar amnesia'.

One of the most important constraints on the age and nature of the progenitor systems is their metallicity $Z$. During stellar evolution, the $\mathrm{C}, \mathrm{N}$, and $\mathrm{O}$ that act as catalysts for the $\mathrm{CNO}$ cycle pile up into ${ }^{14} \mathrm{~N}$, which is converted to ${ }^{22} \mathrm{Ne}$ in the hydrostatic He burning phase through the reactions $\left.{ }^{14} \mathrm{~N}(\alpha, \gamma)\right)^{18} \mathrm{~F}\left(\beta^{+}, \nu_{\mathrm{e}}\right)^{18} \mathrm{O}(\alpha, \gamma){ }^{22} \mathrm{Ne}$. The $\beta^{+}$decay of ${ }^{18} \mathrm{~F}$ increases the neutron excess of the WD material, defined as $\eta=1-2\left\langle Z_{A} / A\right\rangle$ (where $Z_{A}$ is the atomic number and $A$ is the mass number), resulting in a linear scaling of $\eta$ with metallicity: $\eta=0.101 \times$ $Z$ (Timmes et al. 2003). This can have an observable impact on Type Ia supernova spectra (Lentz et al. 2000), but the predicted effects are hard to detect in practice (in one case, SN 2005bl, a progenitor of subsolar metallicity has been suggested by Taubenberger et al. 2008). In this Letter, we describe a new direct method to measure the metallicity of Type Ia SN progenitors using $\mathrm{Mn}$ and $\mathrm{Cr}$ lines from shocked ejecta in the X-ray spectra of SNRs.

\section{THE $M_{M N} / M_{C R}$ RATIO AS A TRACER OF PROGENITOR METALLICITY}

\section{1. $\mathrm{Mn} / \mathrm{Cr}$ vs. $\mathrm{Z}$}

Nuclear burning in Type Ia SNe happens in different regimes depending on the peak temperature reached by the WD material. From higher to lower temperatures, the regimes are: nuclear statistical equilibrium (NSE), incomplete Si burning, incomplete O burning and incomplete C-Ne burning (Woosley 1986). During the explosion itself, electron captures are too slow to change the original value of $\eta$ except in the innermost $\sim 0.2 \mathrm{M}_{\odot}$ of ejecta, where nuclear burning happens at higher densities, leading to neutron-rich NSE (Brachwitz et al. 2000). Outside this region, an imbalance between free neutrons and protons does not affect the production of the most abundant species, with the possible exception of ${ }^{56} \mathrm{Ni}$ at 


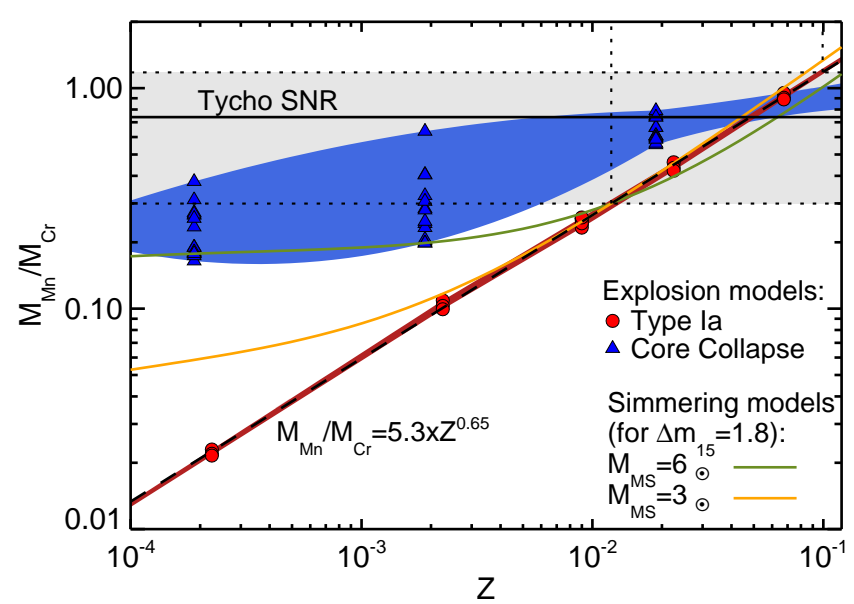

FIG. 1. - Mn to Cr mass ratio as a function of progenitor metallicity for Type Ia (Badenes et al. 2003, 2005, this work) and core collapse (Woosley \& Weaver 1995) SN models, represented with red circles and blue triangles, respectively. The red and blue shaded regions encompass all the models in each class. The dashed line represents a power-law fit to the Type Ia models. The orange and green plots illustrate the impact of weak interactions during the simmering phase for very subluminous Type Ia SNe $\left(\Delta m_{15}=1.8\right)$ in WDs with main sequence progenitor masses of $3 \mathrm{M}_{\odot}$ and $6 \mathrm{M}_{\odot}$, respectively (see $\S 2.2$. The horizontal solid and dotted lines represent the estimated $M_{M n} / M_{C r}$ ratio in the Tycho SNR from the Suzaku observations $(0.74 \pm 0.47$, shaded in gray), which corresponds to $\log (Z)=-1.32_{-0.33}^{+0.67}($ see $\S$ 4).

supersolar metallicities (Timmes et al. 2003). However, some trace nuclei with unequal numbers of protons and neutrons are very efficient at storing the neutron excess. The most abundant among these nuclei is ${ }^{55} \mathrm{Mn}$, which is produced during incomplete Si burning as ${ }^{55}$ Co. When normalized to another product of incomplete Si burning whose nucleosynthesis is insensitive to the neutron excess ( $\mathrm{Cr}$ is the ideal choice), the yield of $\mathrm{Mn}$ becomes an excellent tracer of the progenitor metallicity.

This diagnostic can be quantified with Type Ia SN explosion models. We have used the model grid from Badenes et al. (2003, 2005), complemented with other models calculated with the code described in Bravo et al. (1996). Physical inputs and initial conditions are as in Badenes et al. (2003), except in the cases noted below. We consider examples of several explosion mechanisms: deflagrations, delayed detonations, and pulsating delayed detonations. For the present work, we have recalculated the nucleosynthesis of four delayed detonation models (DDTa, DDTc, DDTe, and DDTf) at different metallicities by altering the value of $X\left({ }^{22} \mathrm{Ne}\right)$ in the preexplosion WD from the canonical 0.01 (corresponding to $\left.Z=9.0 \times 10^{-3}\right)$. We have also calculated one deflagration model starting from a central density twice as large as the other models, and one delayed detonation model with the $\mathrm{C}$ to $\mathrm{O}$ mass ratio $M_{C} / M_{O}=1 / 3$ in the WD instead of the usual 1. In spite of these fundamental differences in explosion mechanisms and initial conditions, we find a very tight correlation between the $\mathrm{Mn}$ to $\mathrm{Cr}$ mass ratio outside the neutron-rich NSE region in the SN ejecta, $M_{M n} / M_{C r}$, and the progenitor metallicity $Z$ (see Figure 11). A power-law fit yields the following relation (with a correlation coefficient $r^{2}=0.9975$ ):

$$
M_{M n} / M_{C r}=5.3 \times Z^{0.65}
$$

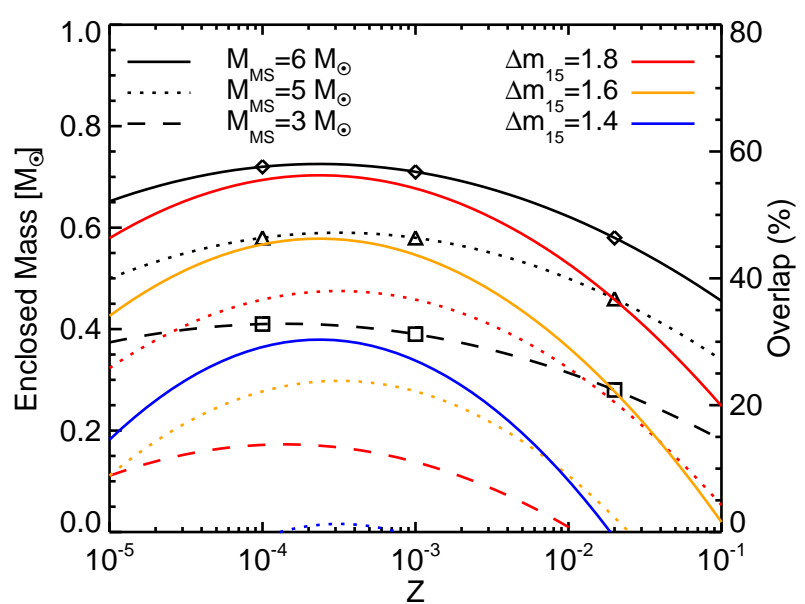

FIG. 2.- Upper limits to $M_{\text {conv }}$ during the simmering phase (left axis, black plots) and its overlap with the Si rich region of ejecta (right axis, colored plots) as a function of $Z$. The black plots represent quadratic interpolations for different $M_{M S}: 3 \mathrm{M}_{\odot}$ (dashed), $5 \mathrm{M}_{\odot}$ (dotted), and $6 \mathrm{M}_{\odot}$ (solid), with the symbols indicating the values from Domínguez et al. (2001) (for $M_{M S}=6 \mathrm{M}_{\odot}$ at $Z=0.02$, we have taken the average between $M_{M S}=7 \mathrm{M}_{\odot}$ and $M_{M S}=5 \mathrm{M}_{\odot}$ ). The colored plots represent the overlap (in $\%)$ between $M_{\text {conv }}$ and the Si-rich region of ejecta, for very subluminous $\left(\Delta m_{15}=1.8\right.$, red $)$, mildly subluminous $\left(\Delta m_{15}=1.6\right.$, orange), and normal, but faint $\left(\Delta m_{15}=1.4\right.$, blue) Type Ia SNe.

Removal of the inner $0.2 \mathrm{M}_{\odot}$ of neutron-rich NSE material from this relation is justified because (1) there is observational evidence that this material does not mix outwards during the explosion (Gerardv et al. 2007; Mazzali et al. 2007) or its aftermath (Fesen et al. 2007); and (2) the dynamical ages of most ejecta-dominated Type Ia SNRs are too small for the reverse shock to have reached so deep into the ejecta. For illustration, we have plotted in Figure 1 the core collapse SN models from Woosley \& Weaver (1995), which also show some correlation between $M_{M n} / M_{C r}$ and $Z$, albeit with a much larger spread and a much shallower slope.

\subsection{The impact of $C$ simmering}

In order for Eq. 1 to hold, the value of $\eta$ set by the progenitor metallicity must remain unchanged between the formation of the WD and the SN explosion. This should be true for DD progenitors if the final merger and runaway happen on dynamical timescales. In slowly accreting WDs, $\eta$ can be modified through electron captures during the so-called 'simmering' phase of non-explosive $\mathrm{C}$ burning that takes place in the $\sim 1000$ yr prior to the explosion (Piro \& Bildsten 2008). The impact of this additional neutronization on the $M_{M n} / M_{C r}$ ratio will depend on the extent of the convective region over which the neutronized material is mixed $\left(M_{\text {conv }}\right)$, and its overlap with the portion of the WD that will undergo incomplete $\mathrm{Si}$ burning where $\mathrm{Mn}$ and $\mathrm{Cr}$ are synthesized.

Some previous studies (Kuhlen et al. 2006; Piro \& Bildsten 2008; Chamulak et al. 2008) have found large values for $M_{\text {conv }}$ by assuming an homogeneous chemical composition for the WD, effectively applying the Schwarzschild criterion for convection. We will adopt the hypothesis that the convective region is limited by the Ledoux criterion to the C-depleted core of the WD created during hydrostatic He-shell burning, $M_{\text {core }}$ (Höflich \& Stein 2002). In the absence of a self-consistent picture for convection inside WDs, 


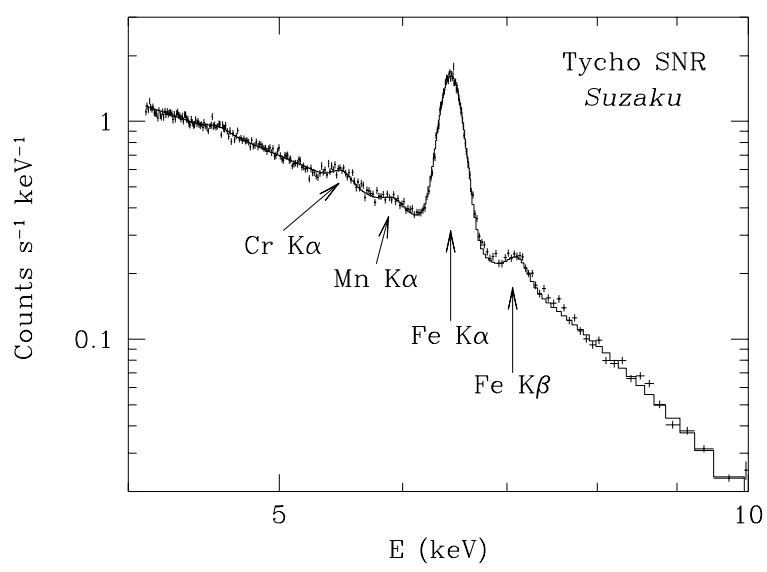

FIG. 3.- X-ray spectrum of the Tycho SNR observed by Suzaku in the vicinity of the $\mathrm{Fe} \mathrm{K} \alpha$ line. The $\mathrm{K} \alpha$ lines of $\mathrm{Cr}$ and $\mathrm{Mn}$ are detected at $>10 \sigma$ and $7 \sigma$ confidence levels, with fluxes of $2.45_{-0.42}^{+0.48} \times 10^{-5}$ and $1.13_{-0.45}^{+0.38} \times 10^{-5}$ photons $\mathrm{cm}^{-2} \mathrm{~s}^{-1}$ respectively (Tamagawa et al. 2008).

this must remain an open issue (see Piro \& Chang 2008, for a discussion), but we believe that our hypothesis is reasonable given the existence of other processes that can reduce the extent of $M_{\text {conv }}$ and limit the mixing of neutronized material. These include the presence of Urca shells (Stein \& Wheeler 2006) and the long thermal diffusion time scale for buoyant bubbles larger than $\sim 100 \mathrm{~m}$ (Garcia-Senz \& Woosley 1995), which prevents them from mixing completely with their surroundings during convection.

According to Mazzali et al. (2007), Si-rich ejecta in Type Ia SNe lie between Lagrangian mass coordinates $1.05 \mathrm{M}_{\odot}$ and $\left(1.55-0.69 \times \Delta m_{15}\right) \mathrm{M}_{\odot}$, where $\Delta m_{15}$ is the light curve width parameter (defined as the decline in blue magnitude 15 days after maximum), which acts as a proxy for SN brightness. In this context, the extent of the overlap between $M_{\text {conv }}$ and the Si-rich ejecta depends on the main sequence mass $M_{M S}$ and initial metallicity of the WD progenitor (which determine $M_{\text {core }}$, Domínguez et al. 2001) and the SN brightness (see Figure 2). This overlap is only significant for subluminous $\left(\Delta m_{15} \geq 1.6\right)$ Type Ia $\mathrm{SNe}$ originated by progenitors with either large $M_{M S}$ or low $Z$, or both. Chamulak et al. (2008) find an upper limit for the increase of $\eta$ during the simmering phase of $\Delta \eta=0.0015$, which is comparable to the value of $\eta$ in solar material. The impact of simmering on the $M_{M n} / M_{C r}$ ratio can then be estimated by mixing material with $M_{M n} / M_{C r}=0.3$ (appropriate for the value of $Z_{\odot}$ derived by Asplund et al. 2005) into the incomplete Si burning region, in a proportion equivalent to the extent of the overlap shown in Figure 2. The green and orange plots in Figure 1 are two examples of such 'simmeringmodified' models for very subluminous $\left(\Delta m_{15}=1.8\right)$ Type Ia SNe, illustrating our conclusion that $\mathrm{C}$ simmering will only modify the $M_{M n} / M_{C r}$ ratio in the SN ejecta for very subluminous $\mathrm{SNe}$, and then only in cases where $M_{M S}$ is large, or $Z$ is low, or both.

\section{MEASURING THE $M_{M N} / M_{C R}$ RATIO IN SNRS}

The work in this Letter is motivated by the recent Suzaku detection of $\mathrm{Mn}$ and $\mathrm{Cr}$ in the X-ray spectrum of the Tycho SN reported by Tamagawa et al. (2008) (see Figure 31). This observational result opens the

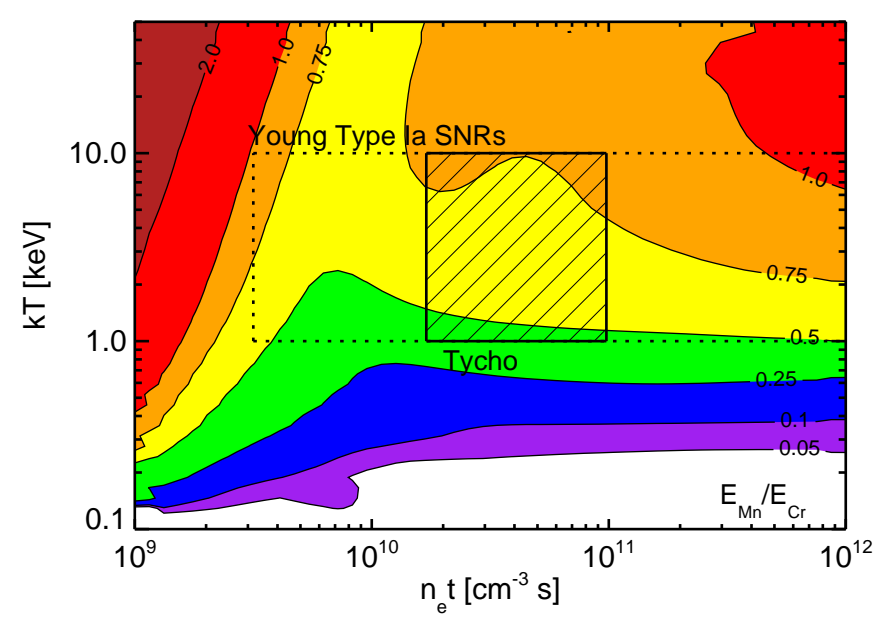

FIG. 4.- Interpolated $\mathrm{Mn}$ to $\mathrm{Cr}$ specific $\mathrm{K} \alpha$ emissivity ratio as a function of $n_{e} t$ and $k T$. The dotted box encompasses the values of $n_{e} t$ and $k T$ found in the Si-rich ejecta of six young Type Ia SNRs by Badenes et al. (2007): $9.49 \leq \log \left(n_{e} t\right) \leq 11.94 \mathrm{~cm}^{-3}$ s; $1.0 \leq k T \leq 10.0 \mathrm{keV}$. The striped area corresponds to the region of parameter space appropriate for the Tycho SNR, $10.23<$ $\log \left(n_{e} t\right) \leq 10.99 \mathrm{~cm}^{-3} \mathrm{~s} ; 1.0 \leq k T \leq 10.0 \mathrm{keV}$.

possibility of studying the $M_{M n} / M_{C r}$ ratio in Type Ia SN ejecta, which cannot be done using optical SN spectra due to the 2.7 yr half-life of ${ }^{55} \mathrm{Fe}$ in the decay chain ${ }^{55} \mathrm{Co} \rightarrow{ }^{55} \mathrm{Fe} \rightarrow{ }^{55} \mathrm{Mn}$. Since $\mathrm{Mn}$ and $\mathrm{Cr}$ are synthesized together in the explosion and have very similar electronic structures, it is possible to estimate their mass ratio from the line flux ratio: $M_{M n} / M_{C r}=$ $1.057 \times\left(F_{M n} / F_{C r}\right) /\left(E_{M n} / E_{C r}\right)$, where 1.057 is the ratio of atomic masses, $F_{M n} / F_{C r}$ is the line flux ratio, and $E_{M n} / E_{C r}$ is the ratio of specific emissivities per ion.

Public data bases for X-ray astronomy do not usually include lines from trace elements like $\mathrm{Mn}$ and $\mathrm{Cr}$, but the value of $E_{M n} / E_{C r}$ can be estimated by interpolation along the atomic number sequence from elements with available data (Hwang et al. 2000). We have used the ATOMDB data base (Smith et al. 2001) to retrieve $\mathrm{K} \alpha$ line emissivities for $\mathrm{Si}, \mathrm{S}, \mathrm{Ar}, \mathrm{Ca}, \mathrm{Fe}$, and $\mathrm{Ni}\left(Z_{A}=14,16,18,20,26,28\right)$ as a function of ionization timescale $n_{e} t$ and electron temperature $k T$, the two variables that control the line emission of a plasma in nonequilibrium ionization. Then we performed a spline interpolation to obtain $E_{M n} / E_{C r}$, which we plot in Figure4, together with the region of the $\left(n_{e} t, k T\right)$ parameter space that is populated by young Type Ia SNRs (see the Appendix in Badenes et al. 2007). The uncertainty in the value of $E_{M n} / E_{C r}$ comes from the variation within this region and the error introduced by the interpolation itself, which we have estimated by comparing the true and interpolated values for the $E_{A r} / E_{S}$ ratio. For the larger region of the $\left(n_{e} t, k T\right)$ plane plotted in Figure 4 , we find $E_{M n} / E_{C r}=0.69 \pm 0.32\left(E_{M n} / E_{C r}=0.66 \pm 0.26\right.$ for the smaller region appropriate for Tycho).

\section{DISCUSSION AND CONCLUSIONS: THE METALLICITY OF TYCHO'S PROGENITOR}

From the Suzaku observation, $F_{M n} / F_{C r}=0.46 \pm 0.21$, which gives $M_{M n} / M_{C r}=0.74 \pm 0.47$. This mass ratio translates into a metallicity of $Z=0.048_{-0.036}^{+0.051}$ for the progenitor of the Tycho SNR $\left(\log (Z)=-1.32_{-0.33}^{+0.67}\right)$. The large error bar in this result is dominated by the statistical uncertainties in the X-ray fluxes from the faint 
Mn and Cr lines, and it will be reduced in an upcoming, approved deeper Suzaku observation. At present, we find a strong indication for a supersolar metallicity $\left(\log \left(Z / Z_{\odot}\right)=0.45_{-0.60}^{+0.31}\right.$ with the solar value from Grevesse \& Sauval (1998); $\log \left(Z / Z_{\odot}\right)=0.60_{-0.60}^{+0.31}$ with the newer value from Asplund et al. (2005)). Our results are compatible with a solar metallicity, but subsolar values can be discarded with confidence. Given this measurement and the fact that Tycho's SN was probably either normal or slightly overluminous (Badenes et al. 2006; Ruiz-Lapuente 2004), we do not have to concern ourselves with the impact of neutronization during $\mathrm{C}$ simmering in this particular case. The Tycho SNR is 59 pc above the Galactic plane at a Galactocentric radius of $9.4 \mathrm{kpc}$ (assuming a distance of $2.4 \mathrm{kpc}$, Smith et al. 1991). A supersolar metallicity is higher than average for this location, but well within the spread of measured $[\mathrm{Fe} / \mathrm{H}]$ values (Nordström et al. 2004). This combination of Galactrocentric radius and metallicity suggests a young progenitor age (a few Gyr or less), which would make Tycho a candidate for the 'prompt' channel of Type Ia $\mathrm{SNe}$, but the scatter in the age-metallicity relations and the uncertainties in our measurement are too large to be more specific on this point.

In this Letter, we have proposed a new method to measure the metallicity of Type Ia SN progenitors using Mn and $\mathrm{Cr}$ lines in the X-ray spectra of their SNRs. We have applied it to the Tycho SNR and obtained a strong indication that its progenitor had a solar or supersolar metallicity. The main strength of our method is its simplicity: it is based on well-known nuclear physics and observable parameters that are easy to measure. Detection of $\mathrm{Mn}$ and $\mathrm{Cr}$ lines in other young Type Ia SNRs in the Galaxy should be possible with Suzaku, Chan$d r a$, and XMM-Newton observations. A few additional SNRs in the Magellanic Clouds might be reached with deep exposures, bringing the total of potential targets to perhaps a dozen. This number would increase significantly with the inclusion of SNRs in nearby galaxies like M31 and M33, which may be accessible to next generation X-ray observatories like Constellation- $X$ and $X E U S$. Even with a small sample of objects, we should be able to use this method to verify and complement the indirect metallicity studies of extragalactic Type Ia SNe (Gallagher et al. 2005; Prieto et al.2008) and test claims about the metallicity dependence of the Type Ia SN rate (Kobavashi et al. 1998). Measurements or upper limits below a certain threshold $\left(M_{M n} / M_{C r} \lesssim 0.1\right)$ would also provide interesting constraints on the extent of the convective region in accreting WDs. We conclude by noting that $\mathrm{Mn}$ and $\mathrm{Cr}$ lines have already been detected by $A S C A$ in the Galactic SNR W49B (Hwang et al. 2000). The measured line flux ratio also suggests a solar or supersolar progenitor metallicity, but both the age and the SN type of W49B are controversial (Hwang et al. 2000; Badenes et al. 2007). We defer a detailed discussion of this object and the application of our method to other Galactic SNRs to a forthcoming publication.

The authors are grateful to Inese Ivans, Bruce Draine, and Jim Stone for discussions. We are also happy to acknowledge the work of the Suzaku Science Working Group and the members of the Suzaku Tycho team. Support for this work was provided by NASA through Chandra Postdoctoral Fellowship Award Number PF6-70046 issued by the Chandra X-ray Observatory Center, which is operated by the Smithsonian Astrophysical Observatory for and on behalf of NASA under contract NAS803060. EB is supported by grants AYA2007-66256 and AYA2005-08013-C03-01. JPH is partially supported by NASA grant NNG05GP87G.

\section{REFERENCES}

Asplund, M., Grevesse, N., \& Sauval, A. J. 2005, in ASP Conf. Ser. 336: Cosmic Abundances as Records of Stellar Evolution and Nucleosynthesis, ed. T. G. Barnes, III \& F. N. Bash, 25

Badenes, C., Borkowski, K. J., \& Bravo, E. 2005, ApJ, 624, 198

Badenes, C., Borkowski, K. J., Hughes, J. P., Hwang, U., \& Bravo, E. 2006, ApJ, 645, 1373

Badenes, C., Bravo, E., Borkowski, K. J., \& Domínguez, I. 2003, ApJ, 593, 358

Badenes, C., Hughes, J. P., Bravo, E., \& Langer, N. 2007, ApJ, 662,472

Brachwitz, F., et al.. 2000, ApJ, 536, 934

Bravo, E., Tornambé, A., Domínguez, I., \& Isern, J. 1996, A\&A, 306, 811

Chamulak, D. A., Brown, E. F., Timmes, F. X., \& Dupczak, K. 2008, ApJ, 677, 160

Domínguez, I., Höflich, P., \& Straniero, O. 2001, ApJ, 557, 279

Fesen, R. A., Höflich, P. A., Hamilton, A. J. S., Hammell, M. C., Gerardy, C. L., Khokhlov, A. M., \& Wheeler, J. C. 2007, ApJ, 658,396

Gallagher, J. S., Garnavich, P. M., Berlind, P., Challis, P., Jha, S., \& Kirshner, R. P. 2005, ApJ, 634, 210

Garcia-Senz, D. \& Woosley, S. E. 1995, ApJ, 454, 895

Gerardy, C. L., et al.. 2007, ApJ, 661, 995

Grevesse, N. \& Sauval, A. J. 1998, Space Science Reviews, 85, 161

Höflich, P. \& Stein, J. 2002, ApJ, 568, 779

Hughes, J. P., Chugai, N., Chevalier, R., Lundqvist, P., \& Schlegel, E. 2007, ApJ, 670, 1260

Hwang, U., Petre, R., \& Hughes, J. P. 2000, ApJ, 532, 970

Ihara, Y., Ozaki, J., Doi, M., Shigeyama, T., Kashikawa, N., Komiyama, K., \& Hattori, T. 2007, PASJ, 59, 811
Kobayashi, C., Tsujimoto, T., Nomoto, K., Hachisu, I., \& Kato, M. 1998, ApJ, 503, L155

Kuhlen, M., Woosley, S. E., \& Glatzmaier, G. A. 2006, ApJ, 640, 407

Lentz, E. J., Baron, E., Branch, D., Hauschildt, P. H., \& Nugent, P. E. 2000, ApJ, 530, 966

Leonard, D. C. 2007, ApJ, 670, 1275

Maoz, D. 2008, MNRAS, 384, 267

Maoz, D. \& Mannucci, F. 2008, arXiv:0801.2898

Mazzali, P. A., Röpke, F. K., Benetti, S., \& Hillebrandt, W. 2007, Science, 315, 825

Nordström, B., Mayor, M., Andersen, J., Holmberg, J., Pont, F., Jørgensen, B. R., Olsen, E. H., Udry, S., \& Mowlavi, N. 2004, A\&A, 418, 989

Panagia, N., Van Dyk, S. D., Weiler, K. W., Sramek, R. A.,

Stockdale, C. J., \& Murata, K. P. 2006, ApJ, 646, 369

Piro, A. L. \& Bildsten, L. 2008, ApJ, 673, 1009

Piro, A. L. \& Chang, P. 2008, ApJ, in press (arXiv:0801.1321)

Prieto, J. L., Stanek, K. Z., \& Beacom, J. F. 2008, ApJ, 673, 999

Roelofs, G., Bassa, C., Voss, R., \& Nelemans, G. 2008, arXiv:0802.2097

Ruiz-Lapuente, P. 2004, ApJ, 612, 357

Ruiz-Lapuente, P., et al.. 2004, Nature, 431, 1069

Scannapieco, E. \& Bildsten, L. 2005, ApJ, 629, L85

Smith, R., Kirshner, R., Blair, W., \& Winkler, P. 1991, ApJ, 375, 652

Smith, R. K., Brickhouse, N. S., Liedahl, D. A., \& Raymond, J. C. 2001, ApJ, 556, L91

Stein, J. \& Wheeler, J. C. 2006, ApJ, 643, 1190

Tamagawa, T., et al.. 2008, PASJ, submitted 
Taubenberger, S., et al.. 2008, MNRAS, 385, 75

Timmes, F. X., Brown, E. F., \& Truran, J. W. 2003, ApJ, 590, L83

Voss, R. \& Nelemans, G. 2008, Nature, 451, 802

Woosley, S. E. 1986, in Saas-Fee Advanced Course 16:

Nucleosynthesis and Chemical Evolution, ed. J. Audouze,

C. Chiosi, \& S. E. Woosley, 1-195
Woosley, S. E. \& Weaver, T. A. 1995, ApJS, 101, 181 